\title{
ENCAPSULAÇÃO DE COMPOSTOS BIOATIVOS DE Bidens Pilosa L. EM PARTÍCULAS LIPÍDICAS SÓLIDAS
}

\author{
T. C. SANT'ANNA, D. F. C. CORTÉS-ROJAS, C. R. F. SOUZA, W. P. OLIVEIRA* \\ ${ }^{1}$ Universidade de São Paulo, Faculdade de Ciências Farmacêuticas de Ribeirão Preto, Laboratório \\ de P\&D em Processos Farmacêuticos - LAPROFAR. \\ *e-mail: wpoliv@fcfrp.usp.br
}

\begin{abstract}
RESUMO
A Bidens pilosa L. (Asteraceae) é uma espécie vegetal conhecida popularmente como picão preto. Esta planta possui diversas atividades biológicas tais como hepatoprotetora, antimicrobiana, antimalárica, antitumoral e antioxidante. Atualmente o desenvolvimento de fitoterápicos está focado na obtenção de formulações que permitam conseguir uma maior estabilidade e melhorem a absorção dos compostos bioativos. As formulações lipídicas têm sido utilizadas com sucesso para o aumento da biodisponibilidade. Por outro lado, a secagem por spray drying é uma alternativa para o aumento da estabilidade. O objetivo deste trabalho foi a secagem de formulações lipídicas sólidas contendo o extrato da $B$. pilosa por spray drying. Foram investigadas a influência da composição da formulação e as condições do processo nas propriedades físicas e químicas dos produtos particulados formados. Lipídeos sólidos (ácido esteárico, cera de abelha, compritol, precirol) e líquidos (óleo de palma, Kollisolv) assim como tensoativos (tween 80 e poloxamer 407) foram utilizados no desenvolvimento de emulsões estáveis como parte dos estudos de pré-formulação. As dispersões líquidas foram caracterizadas visualmente (macroscopicamente) quanto a separação de fases, foi realizado o teste de estabilidade após centrifugação e análise microscópica. Estas análises permitiram selecionar as emulsões mais estáveis às quais foram selecionadas para serem submetidas à secagem por spray dryer. As formulações secas foram caracterizadas quanto à umidade, atividade de água e propriedades de fluidez. A recuperação do produto após a secagem sofreu influencia da composição da formulação. A atividade de água e umidade do produto estão dentro dos limites considerados seguros, favorecendo a estabilidade microbiológica.
\end{abstract}

\section{INTRODUÇÃo}

Atualmente, a necessidade de buscar novas alternativas para o tratamento de inúmeras enfermidades tem contribuído significativamente para o crescimento do uso de produtos derivados de plantas (fitoterápicos). Plantas medicinais e aromáticas são importantes fontes de compostos bioativos de elevado valor terapêutico e nutricional.

A facilidade de acesso aos fitoterápicos é uma das principais razões que justificam sua ampla utilização em países em desenvolvimento e nesse sentido a OMS tem trabalhado para desenvolver e implantar normas, pautas e metodologias que permitam garantir a eficácia, segurança e qualidade.

A produção de extratos secos padronizados é uma alternativa promissora, representando um campo em crescente expansão, já que a tendência atual das indústrias farmacêuticas é a substituição das formas fluídas tradicionais pelo extrato na forma de pó. Dentre as razões a favor dessa tendência, pode-se destacar a maior 
concentração, estabilidade e facilidade de padronização dos princípios ativos presentes nas plantas, facilidade de transporte, menor espaço necessário para o armazenamento do produto e menores riscos de contaminação microbiana, vantagens essas que aumentam o valor agregado do produto (OLIVEIRA e PETROVICK, 2010).

A $B$. pilosa é uma espécie da família Asteraceae, conhecida como picão ou picãopreto. É originária da América tropical, porém amplamente distribuída nas regiões tropicais e subtropicais do mundo (BRANDÃO et al., 1998). Esta espécie vegetal encontra-se na relação de 71 plantas de interesse ao Sistema Único de Saúde (RENISUS) e na Resolução RDC No 10, de 9 de Março de 2010 da Agência Nacional de Vigilância Sanitária (BRASIL, 2010).

A literatura apresenta grande número de estudos experimentais sobre a atividade biológica da $B$. pilosa, destacando-se a atividade antioxidante, hepatoprotetora, antimalárica, antimicrobiana e antitumoral (CORTÉS-ROJAS e OLIVEIRA, 2012). Os principais metabólitos secundários encontrados na planta são os flavonoides e poliacetilenos. $\mathrm{Na}$ Amazônia, infusões e soluções hidroalcoólicas de B. pilosa são consideradas úteis no tratamento da malária e doenças hepáticas. Brandão et al. (1997) mostrou a existência de uma correlação entre a presença de poliacetilenos nas plantas e a atividade antimalárica, sendo descrita a ocorrência de instabilidade desses compostos quando isolados.

A elaboração de produtos farmacêuticos utilizando compostos insolúveis, voláteis ou de baixa estabilidade, representa um desafio tecnológico. Diversas estratégias têm sido propostas para solucionar estes inconvenientes. Entre as alternativas que podem ser utilizadas para preservar as características dos compostos presentes no extrato do material vegetal, destacam-se a encapsulação em lipossomas, microemulsões, micro e nano partículas poliméricas. Além de prolongar o tempo de estocagem de produtos alimentícios e farmacêuticos, estas formulações direcionam o fármaco para regiões específicas, favorecendo sua interação com diferentes sistemas biológicos.

Recentemente os sistemas lipídicos têm mostrado um grande potencial para o aumento da biodisponibilidade de fármacos (MULLER; RADTKE; WISSING, 2002). Dentre os mecanismos relacionados com a sua utilização está o aumento da solubilização do fármaco no trato intestinal pelo estímulo na liberação de lípases e colípases pancreáticas, sais biliares e fosfolipídios (PORTER; TREVASKIS; CHARMAN, 2007).

O desenvolvimento de sistemas lipídicos contendo compostos bioativos de plantas medicinais e secos utilizando a técnica de spray drying é uma nova proposta para o melhoramento da qualidade de formulações de fitoterápicos especialmente quando os compostos bioativos têm baixa solubilidade, baixa estabilidade ou alta volatilidade.

O objetivo deste trabalho foi preparar dispersões lipídicas sólidas contendo compostos bioativos de B. pilosa utilizando a técnica de spray drying. Avaliou-se a influência do lipídeo líquido, lipídeo sólido e do tensoativo na estabilidade da formulação líquida e no desempenho da secagem.

\section{MATERIAL}

\subsection{Matéria prima vegetal}

Amostras de $B$. pilosa na fenofase de floração foram coletadas na região de Ribeirão Preto-SP, dividido o material botânico em raízes, caule e folhas-flores.

\subsection{Tensoativos utilizados em extração micelar}

2.2.1. Polissorbato 80 (Tween 80): tensoativo não-iônico utilizado principalmente como estabilizador de ampla variedade de produtos 
alimentícios, cosméticos e medicamentos. 2.2.2. Poloxamer 188: tensoativo não iônico, hidrofílico amplamente utilizado na composição de cosméticos para a dissolução de ingredientes oleosos em água. Suas moléculas são co-polímeros constituídos por unidades básicas de óxido de etileno, óxido de propileno e óxido de etileno.

\subsection{Lipídeos}

2.3.1 Lipídios sólidos: ácido esteárico TP (Via Farma), cera de abelha (Via Farma), di e triacilgliceróis do ácido behenico (Compritol 888 CG ATO Brasquim) e gliceril di estearato (Precirol ATO 5, Gattefossé)

2.3.2 Lipídios liquidos: Óleo de palma orgânico (Companhia refinadora de Amazônia, lote 29/13) e Kollisolv MCT 70 (BASF).

\subsection{Carreadores de estabilização e secagem}

Maltodextrina Mor Rex 1910 (Corn Products do Brasil).

\section{MÉTODOS}

\subsection{Obtenção do extrato vegetal}

As folhas da planta foram secas em estufa de circulação de ar a $45{ }^{\circ} \mathrm{C}$. Uma vez seco, o material foi pulverizado utilizando moinho de facas Marconi modelo MA 680, utilizando malha $20(833 \mu \mathrm{m})$. O extrato da planta foi obtido por maceração dinâmica utilizando etanol $63 \%$ (v/v) como solvente e relação planta/volume de solvente de 1/10 $(\mathrm{m} / \mathrm{v})$, foi empregada a temperatura controlada de $66{ }^{\circ} \mathrm{C}$ e agitação por 30 minutos. A solução extrativa foi concentrada em evaporador rotativo a $50{ }^{\circ} \mathrm{C}$ até um teor de sólidos de $8 \%$. $\mathrm{O}$ extrato foi liofilizado congelando as amostras a $-20{ }^{\circ} \mathrm{C}$ por $48 \mathrm{~h}$ e posteriormente levados ao freezer a $-80{ }^{\circ} \mathrm{C}$ por $4 \mathrm{~h}$, foi utilizada temperatura de condensação de $-40^{\circ} \mathrm{C}$ por $48 \mathrm{~h}$. A solução extrativa, extrato concentrado e extrato liofilizado de $B$. pilosa foram caracterizados quanto ao teor de sólidos, teor de polifenóis totais, taninos e flavonoides totais.

\subsection{Estudos de pré-formulação}

Estudos de pré-formulação foram realizados avaliando a estabilidade de sistemas lipídicos sem a adição de extrato vegetal. A fase aquosa contendo o tensoativo foi aquecida a $60{ }^{\circ} \mathrm{C}$ e vertida sobre a fase lipídica fundida (lipídeo solido e liquido) e mantida sobre agitação mecânica durante 5 minutos, após esse tempo as formulações foram refrigeradas a $-20{ }^{\circ} \mathrm{C}$ por 10 minutos. A préemulsão obtida foi dispersa por um dispositivo de cisalhamento, o Ultra turrax, nas velocidades de $6000 \mathrm{rpm}, 10000 \mathrm{rpm}$ e 18000 rpm por 30s, 30s e 60s, respectivamente para cada velocidade, contemplando um tempo de homogeneização final de 2 minutos para cada formulação desenvolvida. As formulações obtidas foram mantidas a temperatura ambiente e posteriormente caracterizadas quanto ao aspecto macroscópico (visual), turvação, homogeneidade, precipitação, e separação de fases.

\subsection{Elaboração de formulações lipídicas contendo extrato de $B$. pilosa}

Visando à obtenção dos sistemas microparticulados por spray drying e baseado nos resultados dos ensaios de pré-formulação foram preparadas nove formulações contendo o extrato liofilizado de $B$. pilosa. Foi utilizada a maltodextrina DE10 como adjuvante de secagem. A composição das formulações está apresentada na Tabela 1. As formulações obtidas foram caracterizadas quanto ao aspecto macroscópico (visual), microscópico fazendo diluição 1:4 e observando no microscópio Olympus ${ }^{\circledR}$ - modelo BX60MIV) com aumento de 50 e 500 vezes, e teste de centrifugação durante 15 minutos a 1000, 2500 e $3500 \mathrm{rpm}$ a $25^{\circ} \mathrm{C}$. 
Tabela 1 - Formulações lipídicas contendo o extrato liofilizado de B. pilosa submetidas ao processo de secagem por spray drying.

\begin{tabular}{|c|c|c|c|c|c|c|}
\hline Código & $\begin{array}{l}\text { Lipídio } \\
\text { sólido } \\
\text { ( } 10 \text { g ) }\end{array}$ & $\begin{array}{l}\text { Lipídio } \\
\text { líquido } \\
\text { ( } 3 \text { g ) }\end{array}$ & $\begin{array}{l}\text { Tensoativo } \\
\qquad(2 \mathrm{~g})\end{array}$ & $\begin{array}{c}\text { Extrato } \\
\text { liofilizado } \\
(\mathrm{g})\end{array}$ & $\begin{array}{c}\text { Adjuvante } \\
\text { secagem } \\
(10 \mathrm{~g})\end{array}$ & $\begin{array}{c}\text { Água } \\
\text { ultrapura } \\
(\mathbf{m L})\end{array}$ \\
\hline BpCSLN1 & Compritol & - & Tween & 2 & Malto & 88 \\
\hline BpCNLC2 & Compritol & Kollisolv & Tween & 2 & Malto & 88 \\
\hline BpCNLC3 & Compritol & Óleo de palma & Tween & 2 & Malto & 88 \\
\hline BpPSLN2 & Precirol & - & Tween & 2 & Malto & 88 \\
\hline BpPNLC1 & Precirol & Kollisolv & Tween & 2 & Malto & 88 \\
\hline BpPNLC4 & Precirol & Óleo de palma & Tween & 2 & Malto & 88 \\
\hline BpCNLC1 & Compritol & Kollisolv & Poloxamer & 2 & Malto & 88 \\
\hline BpPSLN1 & Precirol & - & Poloxamer & 2 & Malto & 88 \\
\hline BpPNLC3 & Precirol & Óleo de palma & Poloxamer & 2 & Malto & 88 \\
\hline
\end{tabular}

\subsection{Secagem em spray dryer}

Foi utilizado um spray dryer LABPLANT modelo SD 05. Os parâmetros operacionais do secador foram: Temperatura de entrada do ar de secagem $\left(\mathrm{T}_{\mathrm{ge}}\right)$ : $90^{\circ} \mathrm{C}$; Pressão do ar de atomização $\left(\mathrm{P}_{\mathrm{atm}}\right) 2 \mathrm{kgf} / \mathrm{cm}^{2}$, Vazão do ar de atomização $\left(\mathrm{W}_{\mathrm{atm}}\right) 15 \mathrm{~L} / \mathrm{m}$, Vazão de alimentação do extrato ( $\mathrm{W}_{\text {susp }}$ ) $4 \mathrm{~g} / \mathrm{min}$, Vazão do ar de secagem $\left(\mathrm{W}_{\mathrm{g}}\right)$ $60 \mathrm{~m}^{3} / \mathrm{h}$. Os sistemas microparticulados obtidos foram caracterizados quanto ao teor de umidade (Karl Fischer), atividade de água, densidade bruta e de compactação e índices de fluidez (Índice de Carr e Razão de Hausner).

\subsection{Caracterização física e química dos sistemas microparticulados}

Os sistemas microparticulados obtidos foram caracterizados quanto ao teor de umidade (Karl Fischer), atividade de água, densidade bruta e de compactação e índices de fluidez (Índice de Carr e Razão de Hausner). As metodologias empregadas nestas determinações estão descritas nos itens 3.5.1 a 3.5.4.

\subsubsection{Determinação do teor de umidade}

$\mathrm{O}$ teor de umidade dos produtos particulados foi analisado através da titulação pelo método de Karl Fischer (Methrom 870 KF Titrino Plus). Foram utilizadas amostras de aproximadamente $100 \mathrm{mg}$ para cada determinação. As medidas foram realizadas em triplicata e os resultados expressos em média (desvio padrão).

\subsubsection{Determinação da atividade de água}

A atividade de água $\left(\mathrm{A}_{\mathrm{a}}\right)$ das amostras foi determinada em triplicata no equipamento AquaLab 4 TEV (Decagon, Devices) à temperatura de $25^{\circ} \mathrm{C}$, utilizando o sensor de ponto de orvalho. Este parâmetro está relacionado à umidade do pó e possibilita determinar a quantidade de água livre disponível para o crescimento microbiano e para reatividade química e enzimática.

3.5.3 Densidade aparente e de compactação dos pós e índices de compactação

Um grama de cada uma das dispersões lipídicas secas foi pesado, separadamente, e transferido para proveta de $10 \mathrm{~mL}$. A 
densidade aparente foi determinada pela razão entre o volume $(\mathrm{mL})$ ocupado pelo material particulado e a massa total pesada (gramas). Para a determinação da densidade de compactação, uma amostra de um grama de cada uma das dispersões lipídicas secas foi pesada e transferida para a proveta de $10 \mathrm{~mL}$ e submetida a 1250 quedas em superfície lisa e altura de 3,0 $\pm 0,2 \mathrm{~mm}$ com o auxílio do equipamento Calleva ${ }^{\circledR}$ (Frankfurt, Germany). A densidade de compactação foi calculada pela razão entre o volume em mililitros ocupado pelo material particulado após a compactação e a massa em gramas.

\subsection{4 Índices de compactação dos pós}

Foram determinados através da Razão de Hausner (RH) e Índice de Carr (IC). O cálculo de $\mathrm{RH}$ foi determinado pelo quociente entre a densidade de compactação e a densidade aparente da formulação seca (Equação 1). O IC foi expresso pelo quociente da diferença das densidades aparente e de compactação pela densidade aparente. O IC foi expresso em porcentagem, multiplicandose o valor obtido por 100, conforme apresentado na Equação 2

$$
\begin{aligned}
& R H=\frac{d_{500}}{d_{10}} \\
& I C=\frac{\left(d_{500}-d_{0}\right)}{d_{0}} \times 100
\end{aligned}
$$

Onde: $\mathrm{d}_{0}$ : densidade aparente $\left(\mathrm{g} / \mathrm{cm}^{3}\right), \mathrm{d}_{10}$ : densidade após 10 batidas e $\mathrm{d}_{500}$ : densidade após 500 batidas.

\section{RESULTADOS}

Os estudos de pré-formulação são uma etapa fundamental no desenvolvimento de produtos para uso farmacêutico, nutracêutico ou alimentício. Neste trabalho os estudos de pré-formulação realizados com os diferentes lipídeos e tensoativos permitiram avaliar os sistemas heterodispersos mais estáveis fisicamente. Este processo permite reduzir a quantidade de extrato liofilizado a ser utilizado na detecção de sistemas instáveis mesmo sem a adição do extrato. Uma fez realizada esta pré-seleção o foram preparadas as formulações incorporando $\mathrm{o}$ extrato liofilizado da planta.

$\mathrm{Na}$ Tabela 2 são apresentados os resultados da recuperação de produto após a secagem e da caracterização das partículas sólidas obtidas a partir das dispersões lipídicas contendo o extrato liofilizado da $B$. pilosa. Observa-se que a recuperação de produto está dentro dos valores esperados para este processo (CORTÉS-ROJAS e OLIVEIRA, 2012), principalmente se considerarmos a menor temperatura de transição vítrea dessas composições, que tendem a exibir problemas de aderência na câmara de secagem.

A umidade e atividade de água são critérios comuns na avaliação da susceptibilidade à degradação microbiológica de amostras sólidas e, portanto representa um parâmetro de qualidade. Observa-se que todos os sistemas lipídicos microparticulados apresentaram atividade de água inferior ao limite considerado seguro (aw $\leq 0,5$ ), dificultando o crescimento microbiano (LABUZA e ALTUNAKAR 2007). Os valores de umidade encontram-se na faixa de $2,02 \%$ e $3,5 \%$, sendo um resultado que favorece a estabilidade física, química e também microbiológica dos sistemas particulados obtidos. Tanto a umidade como a atividade de água estão estreitamente relacionadas à temperatura de saída do gás $\left(\mathrm{T}_{\mathrm{gs}}\right)$.

A fluidez dos sistemas particulados pode ser avaliada através da determinação da Razão de Hausner (RH) e no Índice de Carr (IC). Hausner observou que a relação entre a densidade bruta e a densidade aparente relacionava-se com a fricção entre as 
partículas e, portanto poderia ser utilizada para predizer as propriedades dos pós. Dessa forma, demonstrou que pós com baixa fricção, como o caso de pós com tamanhos de partículas elevados, apresentavam uma relação em torno de 1,2 e que, para partículas coesivas com fluxo restrito, este valor seria superior a 1,6 (AULTON, 2005). O IC também é uma medida indireta para avaliar a fluidez dos pós. Segundo esta escala, pós com um índice entre 5 e $15 \%$ são considerados de fluidez excelente, de 12 a $16 \%$ boa, 18 a $21 \%$ escassa, 23 a $35 \%$ pobre, de 35 a $38 \%$ muito pobre e maior do que $40 \%$ extremamente deficiente (AULTON, 2005). Analisando a os resultados do Índice de Carr e Razão Hausner, os produtos secos obtidos podem ser classificados como fluidez escassa ou até muito pobre, evidenciando uma baixa fluidez; comumente observada em produtos desidratados por spray drying. Esse comportamento pode ser atribuído ao pequeno tamanho de partícula obtido associado à baixa umidade, resultando em pós altamente compressíveis, os quais apresentam uma alta fricção e resistência ao escoamento.

\section{CONCLUSÕES}

Os estudos de pré-formulação comparando os lipídios sólidos (ácido esteárico, cera de abelha, compritol, precirol) mostraram que o compritol e o precirol foram os lipídios sólidos que produziram emulsões mais estáveis quando foi utilizando o tensoativo tween 80.

Os sistemas lipídicos microparticulados produzidos, apresentaram atividade de água inferior ao limite considerado seguro (aw $\leq$ $0,5)$ e valores de umidade na faixa de $2,02 \%$ a $3,5 \%$, sendo um resultado que favorece a estabilidade microbiológica dos sistemas particulados.

Os sistemas lipídicos secos apresentaram fluxo escasso a fraco, resultado comumente observado em produtos secos em spray dryer. Esta desvantagem pode ser superada pela adição de adjuvantes de fluidez.

Tabela 2 - Caracterização física das dispersões lipídicas após spray drying

\begin{tabular}{cccccc}
\hline \multirow{2}{*}{ Amostra } & $\begin{array}{c}\text { Recuperação } \\
\text { do produto }\end{array}$ & Umidade & $\begin{array}{c}\text { Atividade de } \\
\text { água }\end{array}$ & $\begin{array}{c}\text { Razão de } \\
\text { Hausner }\end{array}$ & $\begin{array}{c}\text { Índice de } \\
\text { Carr }\end{array}$ \\
\cline { 2 - 6 } & $(\%)$ & $(\%)$ & $(-)$ & $(-)$ & $(\%)$ \\
\hline BPCSLN1 & 60,4 & 3,3 & $0,2966 \pm 0,012$ & 10,5 & 21,9 \\
BPCNLC2 & 56,7 & 2,9 & $0,2750 \pm 0,015$ & 10,8 & 28,2 \\
BPCNLC3 & 58,4 & 2,6 & $0,2604 \pm 0,010$ & 10,7 & 19,0 \\
BPPSLN2 & 36,9 & 2,0 & $0,2544 \pm 0,007$ & 10,8 & 28,2 \\
BPPNLC1 & 50,2 & 2,4 & $0,2353 \pm 0,007$ & 10,8 & 35,1 \\
BPPNLC4 & 48,0 & 2,7 & $0,2959 \pm 0,009$ & 10,7 & 19,0 \\
BPCNLC1 & 47,3 & 3,5 & $0,3053 \pm 0,007$ & 10,7 & 22,0 \\
BPPSLN1 & 44,6 & 3,1 & $0,2886 \pm 0,029$ & 10,7 & 19,0 \\
BPPNLC3 & 51,2 & 2,5 & $0,2988 \pm 0,019$ & 10,5 & 22,0 \\
\hline
\end{tabular}




\section{REFERÊNCIAS}

AULTON, M. E. Delineamento de formas farmacêuticas, Trad. George Ortega et al. 2 edição. Porto Alegre: Artmed, 2005.

BRANDÃO, M. G. L.; NERY, C. G. C.; MAMÃO, M. A. S.; KRETTLI, A. U. "Two methoxylated flavone glycosides from Bidens pilosa", Phytochemistry, v.48, p.397-399, 1998.

BRANDÃO, M. G. L.; KRETTLI, A. U.; SOARES, L. S. R.; NERY, C. G. C.; MARINUZZI, H. C. Antimalarial activity of extracts and fractions from Bidens pilosa and other Bidens species (Asteraceae) correlated with the presence of acetylene and flavonoid compounds. Journal of Ethnopharmacology, v.57, p.131-138, 1997.

BRASIL, Ministério da Saúde. Agência Nacional de Vigilância Sanitária (ANVISA). Resolução - RDC n. 10, de 9 de março de 2010. Notificação de drogas vegetais. Brasília, D.F., 2010.

CORTÉS-ROJAS, D.F.; OLIVEIRA, W.P. Physicochemical properties of phytopharmaceutical preparations as affected by drying methods and carries. Drying Technology, v.30, p. 921-934, 2012.

LABUZA, T.P.; ALTUNAKAR, L., 2007. Water activity in foods: fundamentals and applications. Blackwell Publishing Ltd, Oxford, UK.

MULLER, R. H.; RADTKE, M.; WISSING, S. Nanostructured lipid matrices for improved microencapsulation of drugs. International Journal of Pharmaceutics, v. 242, p.121-128, 2002.
OLIVEIRA, O. W.; PETROVICK, P. R. Secagem por aspersão (spray drying) de extratos vegetais: bases e aplicações. Brazilian Journal of Pharmacognosy, v.20, p. 641-650, 2010.

PORTER, C. J. H.; TREVASKIS, N. L.; CHARMAN, W. N. Lipids and lipid-based formulations: optimizing the oral delivery of lipophilic drugs. Nature Reviews Drug Discovery. v.6, 231-248, 2007.

\section{AGRADECIMENTOS}

Os autores agradecem à Fundação de Amparo à Pesquisa do Estado de São Paulo (FAPESP) pelo apoio financeiro (Auxílios 2012/09890-6; 2012/03427-2 e 2011/10333-1), e ao Conselho Nacional de Desenvolvimento Científico e Tecnológico (CNPq), pela bolsa de Iniciação Científica ao primeiro autor. 